\title{
FOOTBALL IN THE THIRD WAVE OF THE COVID-19 PANDEMIC - CONSIDERATIONS REGARDING COMPETITION ORGANIZATION
}

\author{
Ciprian PANAIT ${ }^{1 *}$, Jeferson COLARES ${ }^{2}$ \\ ${ }^{1}$ National University of Physical Education and Sport, Faculty of Physical Education and Sport, Bucharest, \\ Romania \\ ${ }^{2}$ Federal University of Minas Gerais, School of Physical Education, Physiotherapy and Occupational Therapy, \\ Belo Horizonte, Brazil \\ *Corresponding author: cipi.rapid@yahoo.com
}

https://doi.org/10.35189/dpeskj.2021.60.3.10

\begin{abstract}
In this paper, we aim to complement the experience acquired so far in pandemic times by addressing the current problem of football from certain specific directions, in the conditions of COVID-19 infection. This study used some models (SIR, SEIR and SIRD) that tried to determine the evolution of the pandemic on the basis of a few defining aspects. In a championship involving 20 teams and 38 stages, an officially registered squad for each team can consist of 34 players (30 field players and 4 goalkeepers), and competition organization in the form of a tournament can include: 14 days competition period +7 days break => 4 games scheduled every 3 or 4 days; $10 \times$ 14-day modules with $9 \times 7$-day recovery modules will be required for the competition. The current context is completely new and, in order to train and play during the pandemic, we need to consider the following aspects: the competition system of national football leagues should take the form of the model proposed by us, promoting young players from the academy; the competitions of the national team should take place in the form of tournaments; the referees' activity should be reorganized in relation to the competition system.
\end{abstract}

Keywords: football, training, competition, COVID-19 pandemic.

\section{Introduction}

Football has long crossed the border of a simple sport, becoming the social phenomenon with the greatest representation and impact on people and the policies practised by institutions and governments.

Realising that football can change lives and build destinies, it is appropriate to note the efforts of leaders, players and coaches to complement the objective of achieving on-field performance with that of achieving off-field performance by getting involved in the daily lives of players, spectators and fans. For this reason, we believe that the social responsibility of all those engaged in football should be on another level.

But currently the world is facing the pandemic caused by the SARS-CoV-2 virus, which appeared in China at the end of 2019. Suddenly, without being aware by then of the effects of this medical crisis on the planet, we had to accept a new way of living, working and spending our free time. And when we refer to all the above-mentioned aspects, we refer to a lifestyle, which unfortunately can no longer be as it was.

Historically, religious, musical, sporting and other mass gatherings have been the source of infectious diseases that have spread worldwide (Memish et al., 2019). In this sense, the World Health Organization (WHO) (2020) issued a document stressing that the overall risk related to a mass gathering event is the result of a process that includes: (i) the increased risk 
of COVID-19 transmission associated with the event and an expected strain on health services; (ii) the ability of health authorities and event organizers to prevent and control such risks.

If, in the run-up to the WHO declaration of 11 March 2020 on the pandemic caused by COVID-19, sport in general and football in particular had established very clear training and competition programmes over extended periods, at this time, when the whole world has passed through the second wave and is now on the verge of the third wave of the pandemic, we see the attempts of society and sports federations to crystallise training and competition programmes while observing the health rules aimed at preventing the spread of the virus. However, so far we have not faced a situation where the entire planet must stop. Regarding football, the context in which competition organizers found themselves in the period of isolation was completely new and it is worth mentioning that, even during the Second World War, the official competitions were not completely stopped around the world as happened in the few months of complete isolation. There were also times when different epidemics occurred around major football events: the measles outbreak that took place before the 2008 European Football Championships in Switzerland and in South Africa, in 2009, before the start of the 2010 World Cup, the E-coli endemic outbreak in Germany before the start of the 2011 Women's World Cup, the Dengue, Zika virus and Yellow Fever that emerged during the 2014 World Cup and the 2016 Olympic Games in Brazil. (Duarte Muñoz \& Meyer, 2020)

Therefore, the organizers who went through these experiences stressed the need for a coordinated collaborative response to prevent the spread of infectious diseases with epidemic potential during mass gatherings (Petersen et al., 2016).

At all the above times, the measures taken by relevant institutions managed to mitigate the risks and stop the epidemics, therefore the big football events took place according to the schedule. The experience gained from these major sporting events during which the organizers faced the emergence and re-emergence of several new pathogens with lethal effects on humans has raised awareness of the potential for rapid spread at mass gatherings (Mathis et al., 2015).

In this context, in football, a sport where the contact with the opponent is inevitable, rules and regulations have been developed to allow the gradual return to training and the resumption of official competitions, referring here to national championships and cups. It should be noted that individual training, which was conducted at home by the vast majority of athletes during the quarantine and isolation periods, favoured the resumption of the training programme and games because this individual work prepared athletes for a short pre-season including high-intensity training sessions in wide spaces and good performance with low risk of injury when resuming competitions (Jukic et al., 2020).

Given that mass sporting events have contributed to the spread of SARS-CoV-2, it seems unlikely that major international sporting events with spectators will resume before the population has been immunised or mass vaccination has been achieved (Wackerhage et al., 2020).

Among the first championships that were resumed after the lockdown period in the last season (2019-2020), we can mention the Bundesliga - Germany (May 15, 2020), the Portuguese League (early June 2020), La Liga - Spain and Series A - Italy (mid-June 2020). 
However, in addition to the resumption of official competitions, studies were required on the basis of which to decide whether to continue or stop those competitions. These studies used some models that tried to determine the evolution of the pandemic based on a few defining aspects. Thus, one can talk about the SIR (susceptible-infected-removed) model presented by Kermack and McKendrick (1927), where $S$ represents the number of susceptible people, $I$ is the number of infected people and $R$ is the number of recovered people.

There are other types of models that introduce other components, namely the SEIR models, where $E$ represents the number of exposed people, or SIRD models, where $D$ represents the number of deceased people (Matadi, 2014).

In another approach to the pandemic analysis, Rădulescu et al. (2020) divided participants into age groups and analysed how infected people were framed in each age group. On the basis of this structure, the above authors simulated situations in which different activities and locations were closed in order to determine the effects of the pandemic on different age groups and develop specific measures with an immediate effect on combating the spread of the virus.

However, SIR-like models are not able to predict with certainty the evolution of a pandemic. At best, they can inform on the different probability of trajectories conditioned by specific measures and parameters. (Castro et al., 2020)

Besides these models and variables, there is also a defining element in the pandemic analysis, namely the basic reproduction number, $R$, which is noted as $\left(R_{0}\right)$ and defined as "the average number of secondary cases of an infectious disease arising from a typical case in a totally sensitive population" (Guerra et al., 2017, p. e420).

\section{Topic Addressed}

\section{Management of competition activity}

Based on practical experience in professional football, we aim to approach this topic from a different point of view, specifically that of the coach who must win the match and any competition in which their team will be engaged.

In this respect, certain specific conditions should be simultaneously fulfilled in compliance with the regulations and procedures laid down to combat the spread of SARS-CoV-2.

From a practical point of view, three major situations are identified in which players can contract the virus: from family or friends; from a teammate or other person (technical or medical staff) involved in the training process; from an opponent during an official game. According to Hull et al. (2020), the degree to which contact situations in sport lead to a higher prevalence of SARS-CoV-2 infected people is not clear because no systematic data have been obtained so far.

For all these situations, strict rules have been developed and successfully applied by most clubs involved in competitions that were resumed during the pandemic.

However, it should be noted that the resumption of the preparation process and the subsequent resumption of championships could be achieved in the context of a significant reduction in infection cases as a result of the strict quarantine to which we were subjected for 2-3 months. What we see today in sport and society in general but particularly in football is 
the consequence of this quarantine. In this way, the championships resumed in May or June 2020 could be played on the field, matches could be organized and played in the final stages of European competitions and, last but not least, the matches of national teams could be rescheduled to participate in the final stages for the 2021 European Football Championships, the League of Nations games scheduled for September-November 2020 and the qualifying games for the 2022 FIFA World Cup in Qatar. As it is well known from practice and bibliographical materials, friendly, control and official competitions are an important tool for establishing and maintaining optimal performance (Mujika et al., 2018).

At present, we can say that some experience has been gained in recent months in terms of preparing, organizing and conducting competitions; however, this experience is limited. "There is a big gap in the scientific literature with regard to infection prevention and management in team sports such as football." (Duarte Muñoz \& Meyer, 2020, p. 86)

Therefore, in this paper, we aim to complement the experience acquired so far in pandemic times by addressing the current problem of football from the following directions:

1. Organizing the training of club teams for participation in the 2020-2021 championship;

2. Conducting national championships;

3. Organizing and carrying out the actions of national teams with a view to participate in the competitions mentioned above.

The element of novelty and originality of this paper consists in the fact that these directions of approach refer to the next period in which we notice that the third wave of the pandemic is being felt more and more aggressively. According to the World Health Organization (2020), at the beginning of the first period of relaxation of social restrictions, namely on 27 April 2020, there was an increase of $0.9 \%$ in the number of weekly reported infections, while on 18 May 2020, there was a dramatic increase to $12.33 \%$, reaching $16.45 \%$ on 1 June 2020.

Based on official data presented by the WHO, on 4 May 2020, the number of infected people confirmed in the preceding week was 586,351 with an increase of 5.35\% per week, and at the end of July, 1,809,563 new cases were confirmed with a weekly increase of 3.75\%, which raised the total number of confirmed cases to 19 million people. Unfortunately, this upward trend was maintained, according to official WHO data, reaching 20,439,814 confirmed cases of infection and 744,385 deaths on 14 August 2020 and exponentially higher numbers on 2 October 2020 - 34,495,176 confirmed cases of infection and 1,025,729 deaths.

In recent months, the medical world has highlighted the appearance of virus mutations that make it more aggressive and easier to transmit. In this sense, governments around the world were put in the unpleasant situation of reintroducing partial or total quarantine to limit the spread of these virus mutations.

Our idea of developing a strategy to help football clubs and national teams has become particularly important these days, as the number of infected people is growing rapidly in most countries of the world.

For example, in the official WHO report of 11 April 2021, India reported the highest number of new cases $-873,296 \Rightarrow>$ a $70 \%$ increase, followed by the USA with 468,395 new cases $=>$ a 5\% increase, Brazil with 463,092 new cases $=>$ an $8 \%$ decrease, Turkey with 353,281 new cases $=>$ a $33 \%$ increase and France with 265,444 new cases $=>$ a $9 \%$ increase (World Health Organization, 2021). 
The magnitude of the drama behind these numbers can be more easily observed globally in the picture below (Figure 1):

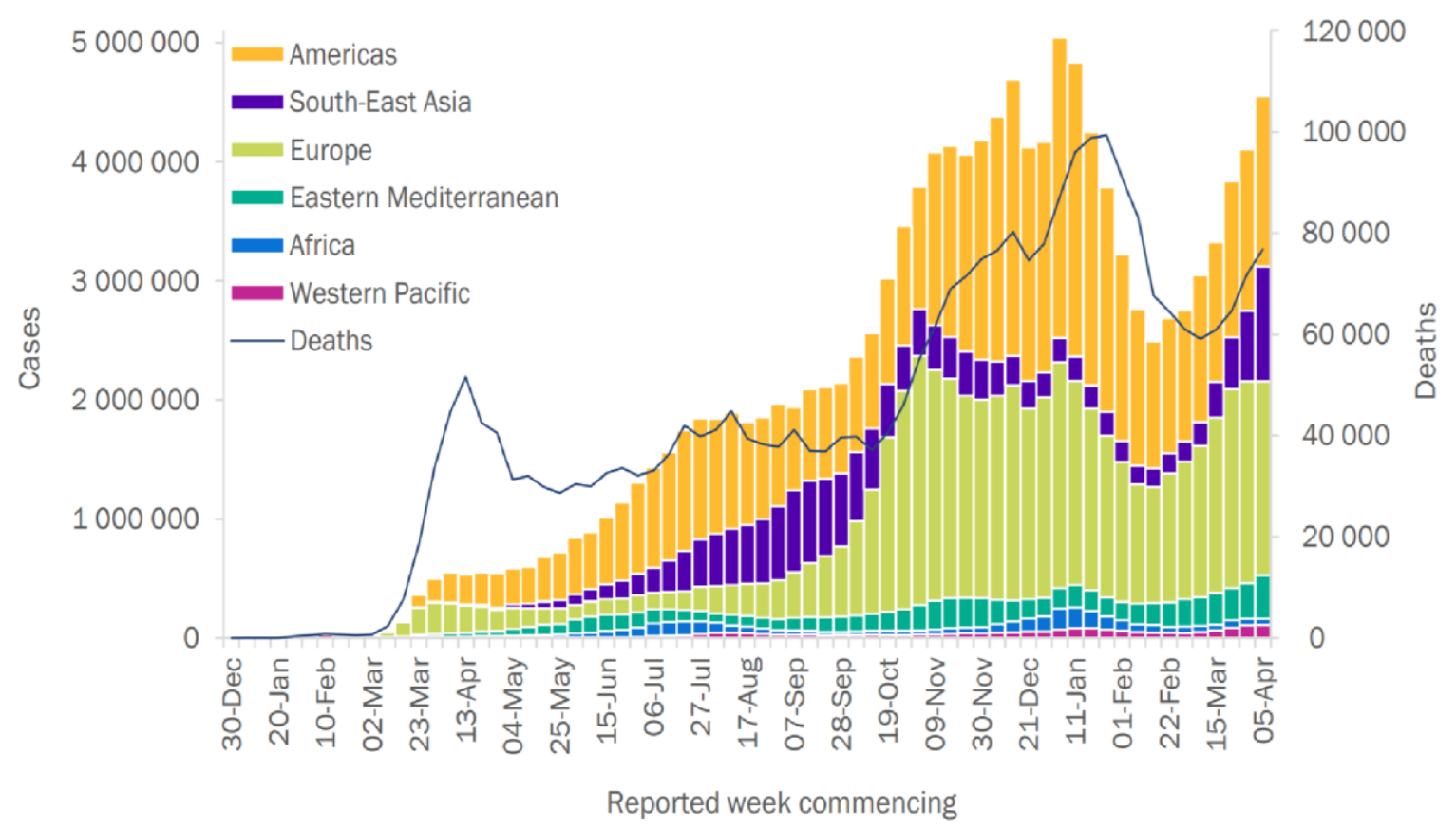

Figure 1. COVID-19 cases reported weekly by WHO Region and global deaths on 11 April 2021 (World Health Organization, 2021, p. 1)

Today, especially in professional football, due to the great pressure put on players, staff and clubs in the light of broad public interests as well as financial constraints, the decision to return to play is of the utmost importance (Scharhag \& Meyer, 2014).

With reference to club teams, we follow with satisfaction and interest the end of the 20192020 national championships establishing the cup winners and naming the best players and coaches, as well as the start and development of the current championships. But nowadays, the great challenge comes only after the resumption of training and the start of the current edition of the national championships, with all the objectives to be achieved. However, some questions arise related to both training and games: under what conditions? when? where? how? with what team? There is uncertainty about compliance with the competition schedule with the primary objective to be reached, namely the organization and development of competitions in health safety conditions, without interruptions caused by the evolution of the pandemic. From the perspective of national teams, we can identify some aspects that need to be clarified: the group of players - selection; training strategy; reducing training time.

Depending on the type and severity of the infectious disease, after healing, maximum exercise intensities can be reached within a period ranging from 3-5 days to 2-3 weeks.

Given the aforementioned issues, we aim to identify some defining aspects that can form a new football strategy for training and competition in the conditions of the third wave of SARS-CoV-2 infection. As a result, we will address separately the defining elements for club teams, national teams and referees. 


\section{Club teams}

The return to regular training and competition will be a slow and complex process; however, the basic strategies that have become crucial in preventing the spread of COVID-19 "should not be forgotten and must continue to be seen as routine precautions, not only for football players but also for the general public" (Duarte Muñoz \& Meyer, 2020, p. 86).

The experience gained in performance football allows us to crystallise several directions to approach the preparation and resumption of official competitions in the new season, under the increasingly obvious conditions of the third wave of the pandemic.

Thus, starting from the experience acquired in the first and second waves of the pandemic, models have been designed that can anticipate the evolution of new virus infections within club teams. A concrete example is the model created by Buldú et al. (2020), who analysed the resumption of the Spanish football league after the lockdown period caused by COVID-19. In their study, $M$ represents the number of teams in the league, $L$ - the maximum number of players registered in each team, Nmatch - the number of days between matches, Ntest - the number of days between tests, Nmatch-1 - the number of days when players trained on the club's grounds.

Based on these variables, a model predicting the infection rate of football players in the current pandemic conditions has been successfully established.

As stated above, the variables of these models largely depend on the evolution of the infection rate. However, given the emergence of the third wave of the pandemic, we consider that these models only have an indicative role because the variables can no longer be anticipated and consequently predictions are risky.

In this situation, we believe that all the conditions and regulations that have made it possible to resume training and competitions are outdated and we propose to carry out the preparation and competition process in a new form that will minimise the risk of infection among players and all those who are involved in training and games.

For the organization of national competitions (championships) in a modular system tournament as the final tournaments of the European or World Championships are organized, the following conditions are imposed: a centralised, unitary training programme under the direct coordination of the technical staff; the possibility to control the variables of the model by organizing the competition for compact periods of time of different lengths, depending on the officially agreed schedule and the degree of fatigue; changing the regulations of national associations to allow the registration of a larger number of players in each team and thus have a sufficient number of players able to cope with the intensive game schedule; dividing the squad into two subgroups that can optimally adapt to the team's level of performance; a reduced number of games for top players, which would represent developments at the highest level and a low risk of injury due to overuse; maintaining the regulatory provision that allows 5 substitutions to be made during an official game so that all players have optimal playing time and can control the effort; effective use of time so that players can participate in the actions of national teams.

For example, in a championship involving 20 teams => 38 stages; the officially registered squad for each team can be 34 players (30 field players and 4 goalkeepers), and competition organization in the form of a tournament can include: 14 days competition period +7 days 
break $=>4$ games scheduled every 3 or 4 days; 10 x 14-day modules with 9 x 7-day recovery modules will be required for the competition. Specifically, players will be divided into two groups of 17, who will be able to participate in the games equally, depending on the decisions of the technical staff. Thus, at the end of the first 4-game module, the coach will decide which group will benefit from recovery and reintegration into social life during the 7 days. The other players will continue their training to participate in the first game of the next 14day module of 4 official games.

The reintegration of players into the training and game programme will occur after the mandatory testing on the 6th day of recovery so that, from the first day of the new 14-day competition module, the tested players are reintegrated into training. Players who are tested positive will follow specific medical protocols.

Advantages of this form of organization and development: low risk of contamination; scheduled training; optimal exercise dosage; recovery control; low risk of injury due to overuse; maintaining a sufficient number of players ready to play at all times. But perhaps the biggest advantage for clubs and national teams is the promotion of the largest possible number of young players raised in training academies. This can quickly translate into financial gains made from the transfer of these young players whose development is accelerated as a result of the conditions listed above.

\section{Referees}

The competition system is the one that determines how the referees' activity will be organized. Therefore, in the aforementioned system, the referees can coordinate their activity and, by reducing the number of days between games, the probability for a referee to be infected will decrease proportionally, taking into account the observance of the testing programme.

We can say that the process of training referees can become a professional one if the same system of organizing training and playing times (camp, training, tests, games, etc.) is observed.

Under these conditions, each national association, depending on the referees' status, can decide to form a group of referees who will remain in a location (camp) to continue training under strict control of compliance with the rules against the spread of SARS-CoV-2.

\section{National teams}

If the amount of bibliographical material on the problem of football during the pandemic, from the point of view of clubs, is reduced, we can say that, in terms of organizing the activity of national teams, bibliographical sources are completely missing.

This issue is all the more important as last autumn the play-off games were scheduled to qualify for the 2021 European Championships and the League of Nations games. In the competition programme presented by us, the actions of national teams should be integrated so as to allow playing games safely with all players while eliminating the risk of infection. 
Consequently, following the evolution of the pandemic, we must be prepared to organize the actions of national teams in the context of the third wave of the pandemic.

According to the draw, the play-off to qualify for the European Championships took place in two rounds as follows: 08.10.2020 - play-off semi-finals; 12.11.2020 - play-off finals (UEFA, 2020), and the scheduling of the Nations League games has already been decided. We will refer below to the qualifying games for the 2022 World Championships in Qatar. The teams participating in this competition are divided into 5 groups of 5 teams and 5 groups of 6 teams each (FIFA, 2021).

Given the increasingly difficult conditions caused by the possible occurrence of the third wave of the pandemic, we believe that the current competition system is outdated and we propose to rethink it in order to reduce the risks to which all participants in these games are exposed. As mentioned before, the actions of national teams should be integrated into the competition programme of club teams as proposed by us. In this way, there should be a lower number of dates in the competition calendar to allow the qualifying games for the final tournament of the 2022 World Championships in Qatar to take place.

Therefore, we propose to reorganize the programme of this competition as follows: playing the games in the form of tournaments; each team in the group will play two qualifying tournaments of 4 or 5 games, depending on the number of teams in the group; each group will play the games from the two qualifying tournaments in two different cities, one for each of the two tournaments; the games will be scheduled every 3 and 4 days, respectively (during a single period); so, instead of the 3 official stages so far where each team played two games every 3 days, the group stage can be completed safely and with minimal risk of infection in reduced time; the first two teams ranked in the group after the two preliminary tournaments will qualify for the final FIFA tournament of the 2022 World Cup in Qatar.

\section{Conclusion}

With the passage of time and the introduction of relaxation measures, we are witnessing a sharp increase in the number of people infected with the new SARS-CoV-2, which, according to experts, can be defined as the third wave of the pandemic. In this context, we believe that all the measures and rules applied since the appearance and spread of the virus until now are no longer enough or no longer observed, which is why, in order to safely continue and end the football competition in the 2020-2021 season, we should rethink and reorganize both the actions of national teams and the referees' activity.

From the increasingly likely perspective of the emergence of the third wave of COVID-19, we consider that the following measures are necessary: playing national football championships in the form of tournaments for compact periods of time in which players must remain in the centralised system to minimise the risk of infection; increasing the number of team players to allow an increased number of games to be played within a very short time, with matches scheduled every 3 or 4 days; alternating competitive periods with short break periods during which players can reintegrate into family and social life; player testing every two days during break periods, with the last test being administered two days before resuming the competition period; maintaining the regulatory provision that allows 5 substitutions to be made during an official game; reorganizing the referees' activity in 
relation to the competition system; considering the referees' team as the club's team and consequently applying the same training and game participation programme as that of club teams, namely with compact periods of centralised training and limitation of interactions with family and the social environment and periods of break and social reintegration.

We believe that the ideas presented in this paper complement the experience acquired during the pandemic period and represent a real basis for action in the next period, in the context of the emergence of the third wave of SARS-CoV-2 infection. However, "even in the event of apparent elimination, SARS-CoV-2 surveillance should be maintained because a resurgence in contagion could be possible as late as 2024" (Kissler et al., 2020, p. 860).

\section{References}

Buldú, J. M., Antequera, D. R., \& Aguirre, J. (2020). The resumption of sports competitions after COVID-19 lockdown: The case of the Spanish football league. Chaos, Solitons \& Fractals, 138: 109964. https://doi.org/10.1016/j.chaos.2020.109964

Castro, M., Ares, S., Cuesta, J. A., \& Manrubia, S. (2020). Predictability: Can the turning point and end of an expanding epidemic be precisely forecast while the epidemic is still spreading? PNAS, 117(42), 261290-26196. https://doi.org/10.1073/pnas.2007868117

Duarte Muñoz, M., \& Meyer, T. (2020). Infectious diseases and football - lessons not only from COVID-19. Science and Medicine in Football, 4(2), 85-86. https://doi.org/10.1080/24733938.2020.1749422

FIFA.com. (2021). UEFA preliminary competition for the FIFA World Cup 2022 ${ }^{T M}$ : Draw procedures. https://resources.fifa.com/image/upload/draw-procedures-for-fifa-world-cupqatar-2022tm-preliminary-draw-uefa.pdf?cloudid=ijoruwm3ly5kgz9wbu6j

Guerra, F. M., Bolotin, S., Lim, G., Heffernan, J., Deeks, S. L., Li, Y., \& Crowcroft, N. S. (2017). The basic reproduction number $\left(\mathrm{R}_{0}\right)$ of measles: A systematic review. The Lancet Infectious Diseases, 17(12), e420-e428. https://doi.org/10.1016/s1473-3099(17)30307-9

Hull, J. H., Loosemore, M., \& Schwellnus, M. (2020). Respiratory health in athletes: Facing the COVID-19 challenge. The Lancet: Respiratory Medicine, 8(6), 557-558. https://doi.org/10.1016/s2213-2600(20)30175-2

Jukic, I., Calleja-González, J., Cos, F., Cuzzolin, F., Olmo, J., Terrados, N., Njaradi, N., Sassi, R., Requena, B., Milanovic, L., Krakan, I., Chatzichristos, K., \& Alcaraz, P. E. (2020). Strategies and solutions for team sports athletes in isolation due to COVID-19. Sports, 8(4): 56. https://doi.org/10.3390/sports8040056

Kermack, W. O., \& McKendrick, A. G. (1927). A contribution to the mathematical theory of epidemics. Proceedings of the Royal Society A: Mathematical, Physical and Engineering Sciences, 115(772), 700-721. https://doi.org/10.1098/rspa.1927.0118

Kissler, S. M., Tedijanto, C., Goldstein, E., Grad, Y. H., \& Lipsitch, M. (2020). Projecting the transmission dynamics of SARS-CoV-2 through the postpandemic period. Science, 268(6493), 860-868. https://doi.org/10.1126/science.abb5793

Matadi, M. B. (2014). The SIRD epidemial model. Far East Journal of Applied Mathematics, 89(1), 1-14.

Mathis, M., Briand, S., \& Prentice, T. (2015). Emerging and re-emerging infectious threats in the 21st century. Weekly Epidemiological Record, 90(20), 238-244. PMID: 25980037

Memish, Z. A., Steffen, R., White, P., Dar, O., Azhar, E. I., Sharma, A., \& Zumla, A. (2019). Mass gatherings medicine: Public health issues arising from mass gathering religious and sporting events. The Lancet, 393(10185), 2073-2084.

https://doi.org/10.1016/S0140-6736(19)30501-X 
Mujika, I., Halson, S., Burke, L. M., Balagué, G., \& Farrow, D. (2018). An integrated, multifactorial approach to periodization for optimal performance in individual and team sports. International Journal of Sports Physiology and Performance, 13(5), 538-561. https://doi.org/10.1123/ijspp.2018-0093

Petersen, E., Wilson, M. E., Touch, S., McCloskey, B., Mwaba, P., Bates, M., Dar, O., Mattes, F., Kidd, M., Ippolito, G., Azhar, E. I., \& Zumla, A. (2016). Rapid spread of Zika virus in the Americas: Implications for public health preparedness for mass gatherings at the 2016 Brazil Olympic Games. International Journal of Infectious Diseases, 44, 11-15. https://doi.org/10.1016/j.ijid.2016.02.001

Rădulescu, A., Williams, C., \& Cavanagh, K. (2020). Management strategies in a SEIR-type model of COVID 19 community spread. Scientific Reports, 10(21256). https://doi.org/10.1038/s41598-020-77628-4

Scharhag, J., \& Meyer, T. (2014). Return to play after acute infectious disease in football players. Journal of Sports Science, 32(13), 1237-1242. https://doi.org/10.1080/02640414.2014.898861

UEFA. (2020). Euro 2020 play-offs: How they worked. https://www.uefa.com/uefaeuro-2020/news/0259-0e6b478eab44-79a1a79706e0-1000-play-offs-all-you-need-to-know/?iv=true

Wackerhage, H., Everett, R., Krüger, K., Murgia, M., Simon, P., Gehlert, S., Neuberger, E., Baumert, P., \& Schönfelder, M. (2020). Sport, exercise and COVID-19, the disease caused by the SARS-CoV-2 coronavirus. German Journal of Sports Medicine, 71(5), E1-E12. doi: $10.5960 / \mathrm{dzsm} .2020 .441$

World Health Organization. (2021). COVID-19 weekly epidemiological update. https://www.who.int/docs/default-source/coronaviruse/situationreports/20210413_weekly_epi_update_35.pdf

World Health Organization. (2020). Key planning considerations for mass gatherings in the context of COVID-19 2020. https://www.who.int/publications/i/item/10665-332235 